ISSN 0103-5150

Fisioter. Mov., Curitiba, v. 24, n. 1, p. 173-180, jan./mar. 2011 Licenciado sob uma Licença Creative Commons

\title{
A produção de conhecimento em fisioterapia: análise de periódicos nacionais (1996 a 2009)
}

\author{
The production of knowledge in physiotherapy: national periodicals \\ analysis (1996-2009)
}

\section{Janeisa Franck Virtuoso ${ }^{[a]}$, Alessandro Haupenthal ${ }^{[b]}$, Natália Duarte Pereira ${ }^{[c]}$, Caroline Pereira Martins $^{[\mathrm{d}]}$, Rodrigo José Knabben ${ }^{[\mathrm{e}]}$, Alexandro Andrade ${ }^{[\mathrm{f}] 1}$}

[a] Mestranda em Ciências do Movimento Humano pela Universidade do Estado de Santa Catarina (UDESC), Florianópolis, SC - Brasil, e-mail: janeisav@yahoo.com.br

[b] Doutorando em Ciências do Movimento Humano pela Universidade do Estado de Santa Catarina (UDESC), Florianópolis, SC - Brasil, e-mail: dedsnet@yahoo.com.br

[c] Mestrando em Ciências do Movimento Humano pela Universidade do Estado de Santa Catarina (UDESC), Florianópolis, SC - Brasil, e-mail: nat_duarte@yahoo.com.br

[d] Mestrando em Ciências do Movimento Humano pela Universidade do Estado de Santa Catarina (UDESC), Florianópolis, SC - Brasil, e-mail: czinha21@gmail.com

[e] Mestrando em Ciências do Movimento Humano pela Universidade do Estado de Santa Catarina (UDESC), Florianópolis, SC - Brasil, e-mail: rodrigokfisio@gmail.com

[f] Professor da Pós-Graduação em Ciências do Movimento Humano da Universidade do Estado de Santa Catarina (UDESC), Florianópolis, SC - Brasil, e-mail: d2aa@udesc.br

\section{Resumo}

Introdução: Avaliar a produção do conhecimento de uma área pode facilitar a compreensão de sua identidade, bem como apontar quais rumos está percorrendo. Objetivo: Verificar qual a área de estudos e os descritores de maior ocorrência em artigos publicados em revistas de circulação nacional voltadas à fisioterapia. Método: Trata-se de uma pesquisa documental com abordagem qualitativa e quantitativa dos dados por meio da análise de conteúdo e da contagem das aparições dos descritores. Foram incluídos resumos de periódicos de fisioterapia brasileiros, com classificação maior ou igual a B2 no WebQualis e acesso livre em

1 Janeisa Franck Virtuoso, Alessandro Haupenthal, Natália Duarte Pereira, Caroline Pereira Martins e Rodrigo José Knabben foram responsáveis pela produção do texto e Alexandro Andrade pela orientação e revisão do artigo. 
sítio da internet. Com esses critérios foram selecionadas a Revista Brasileira de Fisioterapia e a Fisioterapia em Movimento, de 1996 a 2009. Os itens analisados foram: palavras-chave e área de estudo. Utilizou-se o programa TextStat para a contagem das palavras. Resultados: Foram analisados 726 artigos. As áreas de estudo de maior ocorrência foram ortopedia, neurologia, pneumologia e pediatria, que somadas englobam $65 \%$ da produção em fisioterapia, sendo a ortopedia, isoladamente, responsável por 36\% dos estudos. Em relação à palavra-chave, a prevalência foi maior do descritor "fisioterapia", seguido de "idoso" e "atividade física". Discussão: Quanto à área de estudos, o destaque para a ortopedia reflete a atuação do fisioterapeuta, que historicamente concentrou-se em hospitais e clínicas de reabilitação sob a tutela dos médicos ortopedistas, buscando sua independência num segundo momento. Em relação aos descritores, a maior frequência da palavra "fisioterapia" deve ser reavaliada pelos pesquisadores, pois não reflete o conteúdo dos estudos. Conclusão: A produção científica em ortopedia teve maior ocorrência, refletindo a maior área de atuação profissional.

Palavras-chave: Conhecimento. Publicações de divulgação científica. Pesquisa. Fisioterapia.

\section{Abstract}

Introduction: To evaluate the production of knowledge from one area may facilitate the understanding of their identity as well as pointing out which direction is traveling. Objective: To determine which area of studies and greater occurrence of descriptors in articles published in magazines at physiotherapy in periodicals of national circulation. Method: This is a documental research with a qualitative approach through content analysis. We included abstracts from journals of physiotherapy Brazilians, ranked higher or equal to B2 in WebQualis and free access to the website. With these criteria was selected: Brazilian Journal of Physical Therapy and Physical Therapy in Movement, from 1996 to 2009. The items discussed were: keywords and study area. We used the program to count words TextStat. Results: We analyzed 726 articles. Areas of study occurred more frequently orthopedics, neurology, pulmonology and pediatrics, which together comprise 65\% of production in physical therapy, and orthopedics, alone accounts for $36 \%$ of the studies. In relation to the keyword, the prevalence was highest descriptor physiotherapy, followed by elderly and physical activity. Discussion: As for area studies, especially considering the practice of orthopedics physical therapist that has historically focused on hospitals and rehabilitation clinics under the supervision of the orthopedic doctors and seeking its independence a second time. In relation to the descriptors, the highest frequency of the word therapy should be reassessed by the researchers, because it reflects the content of studies. Conclusion: The scientific production in orthopedics was more frequent, reflecting the largest professional performance.

Keywords: Knowledge. Publications for science diffusion. Research. Physiotherapy.

\section{Introdução}

A ampliação da produção do conhecimento no Brasil tem levado diferentes áreas a realizar um balanço dos estudos e pesquisas que estão sendo desenvolvidos por considerarem a importância de tal análise no processo de construção da memória e no aperfeiçoamento do conhecimento (1).

A análise de periódicos científicos tem sido uma modalidade de investigação, com presença significativa e reiterada na literatura, voltada ao estudo da produção de conhecimento (2). Conhecer a produção do conhecimento de uma área pode facilitar a compreensão de sua identidade, bem como apontar perspectivas futuras (3).

A fisioterapia, apesar de comemorar 40 anos de regulamentação e, portanto, ainda muito jovem em relação a outras profissões da saúde, tem avançado na produção de conhecimento específico e na incorporação desses resultados na prática clínica (3-5).

Para Noronem e Widstrom (6), o debate em torno dos pressupostos filosóficos e teóricos para o desenvolvimento relevante da prática e da pesquisa em fisioterapia tem sido encorajado a fim de definir sua identidade e entender a cultura da profissão, fazendo-se necessárias informações sobre seu 
desenvolvimento ao longo do tempo, bem como do conhecimento que produz.

A publicação de resultados de pesquisas científicas tem sido o recurso mais utilizado para legitimar e registrar o avanço do conhecimento, sendo o periódico o meio mais utilizado para esse tipo de divulgação (7).

Diante do exposto, torna-se oportuno apontar os focos de atenção da fisioterapia, no âmbito nacional, ao longo dos últimos anos. Dessa forma, o objetivo do presente estudo foi verificar qual área de estudos e descritores são de maior ocorrência em artigos publicados em revistas de circulação nacional voltadas à fisioterapia.

\section{Método}

Tipo de estudo

Trata-se de uma pesquisa documental com abordagem qualitativa dos dados (8), por meio da análise de conteúdo, do tipo categorial.

\section{Procedimentos}

Assim como nas revisões sistemáticas, os "sujeitos" da investigação (unidade de análise) são os estudos primários selecionados por meio de um método sistemático e predefinido. Os estudos primários podem ser ensaios clínicos aleatórios, estudos de acurácia, estudos de coortes ou qualquer outro tipo de estudo.

A busca pelos artigos foi realizada nas bases de dados eletrônicas a partir dos seguintes critérios de inclusão: periódicos de circulação nacional na área de fisioterapia, com classificação maior ou igual a B2 no WebQualis e disponibilidade livre em algum sítio da internet ou a partir de base de dados como LILACS (Literatura Latino-Americana e do Caribe em Ciências da Saúde) e SciELO (Scientific Electronic Library Online). A partir desses critérios foram selecionadas a Revista Brasileira de Fisioterapia e a Fisioterapia em Movimento.

Os limites desta busca foram dados a partir da disponibilidade dos sítios da internet de gerar os dados necessários para a análise dos artigos. Para a Revista Brasileira de Fisioterapia, o limite foi de 1996 até a data de realização do estudo em 2009 e para a Fisioterapia em Movimento o limite foi de 2005 a 2009.
A análise dos estudos foi realizada posteriormente à coleta de dados dos artigos selecionados e à leitura do resumo. Nesta coleta de dados foi preenchido um instrumento que posteriormente foi utilizado para a análise com o programa específico. Nesse instrumento consta o preenchimento dos seguintes itens: identificação, título do artigo, periódico, ano, autores, palavras-chave e área de estudo.

Para o preenchimento do instrumento, os artigos foram classificados quanto à área de estudos: ortopedia, reumatologia, pneumologia, cardiologia, pediatria, neurologia, uroginecologia, biomecânica, termoeletroterapia e saúde pública. Adotou-se a denominação "outros" quando a categoria da disciplina não somava mais de 5 no total dos artigos. Para análise de frequência dos dados, utilizou-se o programa TextStat (Simple Text Analysis Tool), versão 2.7. Por meio desse software, é possível contabilizar o número de vezes que uma palavra aparece no arquivo, além das relações entre as áreas de estudo e as palavras-chave.

\section{Resultados}

Foram registrados 726 artigos, cuja distribuição bienal apresenta-se na Figura 1. É possível observar que até $2002 / 2003$ a publicação em fisioterapia teve menor expressão. Isso se deve ao fato de que nesse período foram selecionadas somente publicações da Revista Brasileira de Fisioterapia. A partir de 2005, com a disponibilidade também das publicações pela Fisioterapia em Movimento, houve um importante aumento no número de artigos.
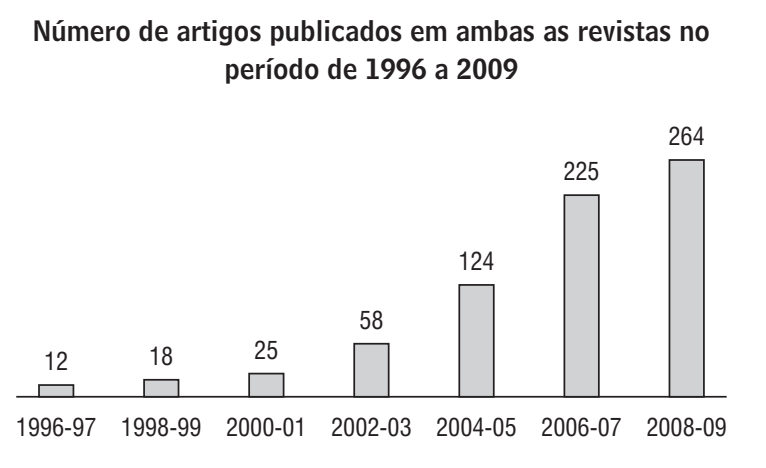

Figura 1 - Número de artigos publicados na Revista Brasileira de Fisioterapia e Fisioterapia em Movimento no período de 1996 a 2009 apresentados em períodos de dois anos 
Conforme pode ser observado na Tabela 1 , a ortopedia foi a área de estudos mais prevalente $(36,2 \%)$, seguida por pneumologia $(11,4 \%)$ e neurologia $(9,7 \%)$. As disciplinas menos prevalentes foram reumatologia $(3,0 \%)$, uroginecologia $(2,7 \%)$ e saúde pública $(1,9 \%)$. A categoria "outros" apresentou frequência de $7,6 \%$ e foi composta por artigos sobre ética em pesquisa ou fisioterapia baseada em evidências.

Tabela 1 - Frequência (n) e porcentagem (\%) das disciplinas vinculadas aos artigos publicados na Revista Brasileira de Fisioterapia e na Fisioterapia em Movimento

\begin{tabular}{lcc}
\hline Disciplina & Frequência (n) & Porcentagem (\%) \\
\hline Ortopedia & 263 & 36,2 \\
Pneumologia & 83 & 11,4 \\
Neurologia & 71 & 9,7 \\
Pediatria & 66 & 9,1 \\
Outros & 55 & 7,6 \\
Termoeletroterapia & 53 & 7,3 \\
Cardiologia & 42 & 5,8 \\
Biomecânica & 37 & 5,1 \\
Reumatologia & 22 & 3,0 \\
Uroginecologia & 20 & 2,7 \\
Saúde pública & 14 & 1,9 \\
\hline
\end{tabular}

As disciplinas mais frequentes foram analisadas segundo o período de publicação, conforme pode ser observado na Figura 2. Foi selecionado o período entre 2005 e 2009 por contemplar publicações de ambas as revistas selecionadas e analisar o período em que ocorreu aumento no número de publicações (2005). Por meio desse gráfico, nota-se a predominância de estudos vinculados à área ortopédica e a ausência de outra área de estudo em destaque.

Após a contagem das palavras-chave de ambos os periódicos, foram selecionados 13 descritores cuja frequência de aparição foi maior que 25 vezes nos 726 artigos pesquisados. Ao analisar as palavras-chave mais frequentes, foi possível observar que o descritor "fisioterapia" foi o mais utilizado nos artigos científicos em ambas as revistas $(21,5 \%$ na revista Fisioterapia em Movimento e 14,2\% na Revista Brasileira de Fisioterapia). Chama também atenção a alta frequência da palavra idosa $(6,9 \%$ e $8,1 \%$, respectivamente).

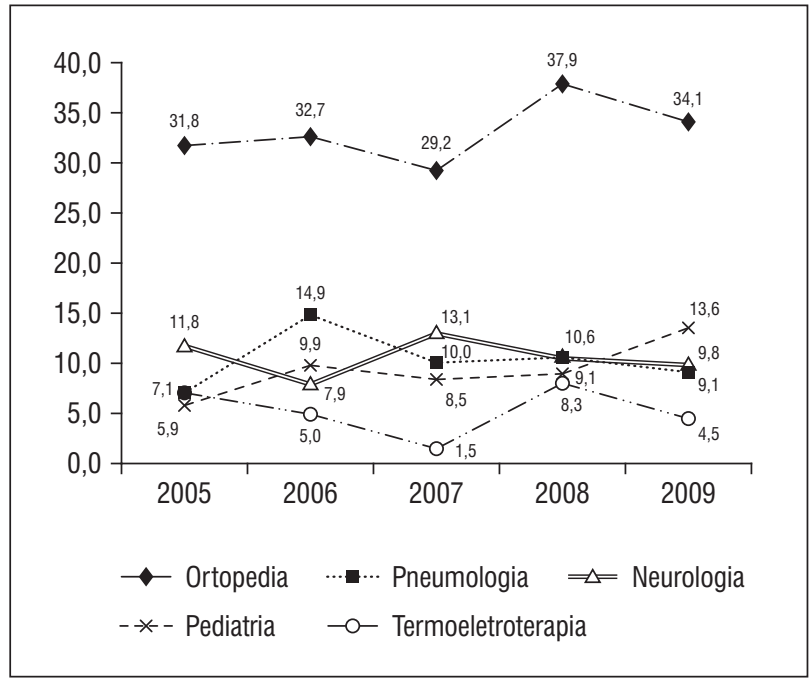

Figura 2 - Distribuição (\%) das disciplinas mais frequentes no período de 2005 a 2009

Ao analisar a distribuição das palavras-chave segundo o periódico e a área de estudo, observa-se que "ortopedia" aparece vinculada a quase todas as palavras-chave, com exceção dos descritores "qualidade de vida" e "crianças" na Fisioterapia em Movimento. Destaca-se ainda o descritor "estimulação elétrica", muito vinculado à disciplina de ortopedia (Figura 3).

\section{Discussão}

Segundo Costa e Nascimento (9), ao longo da última década a pós-graduação desempenhou importante papel na melhoria da produção intelectual brasileira, tanto quantitativa quanto qualitativamente. $\mathrm{O}$ aumento no número de publicações ao longo dos anos, como apresentado neste estudo, pode justificar-se pelo número crescente de pesquisadores doutores com graduação em fisioterapia na última década. Segundo Coury e Vilella (4), em 1998 existiam 57 fisioterapeutas doutores, enquanto em 2008 houve um aumento para 573, demonstrando um grande esforço por capacitação científica realizado pela comunidade de fisioterapeutas.

Segundo Caldas (10), a partir da década de 90 é que a atitude de buscar embasamentos científicos para a intervenção passa a ser realizada. No entanto, essa busca ainda está mais concentrada nas atividades de pesquisa dos docentes de algumas escolas brasileiras, em especial nas universidades públicas (Universidade Federal de São Carlos - UFSCar, 
Universidade de São Paulo - USP e Universidade Federal de Minas Gerais - UFMG, por exemplo), onde existe a maior relação de professores mestres e doutores, além de estímulo permanente às atividades de pesquisa e ao desenvolvimento de programas de pós-graduação stricto sensu.

A política de divulgação do conhecimento científico parece, também, não estar sendo valorizada. A palavra-chave mais frequente neste estudo foi fisioterapia, cujo descritor é muito amplo e nada reflete sobre quais rumos estão sendo percorridos pela área. Os autores, ao submeterem um artigo, precisam estar cientes de que tanto a Revista Brasileira de Fisioterapia quanto a Fisioterapia em Movimento são vitrines da produção científica nacional e, portanto, precisam divulgar com precisão seus resultados, desde as palavras-chave até a conclusão. Acredita-se que o uso frequente desse descritor, que pouco reflete a especificidade da área, relaciona-se à valorização técnica da profissão. No entanto, essa situação parece estar sendo alterada graças ao aumento de estudos relacionados à "confiabilidade e validade".

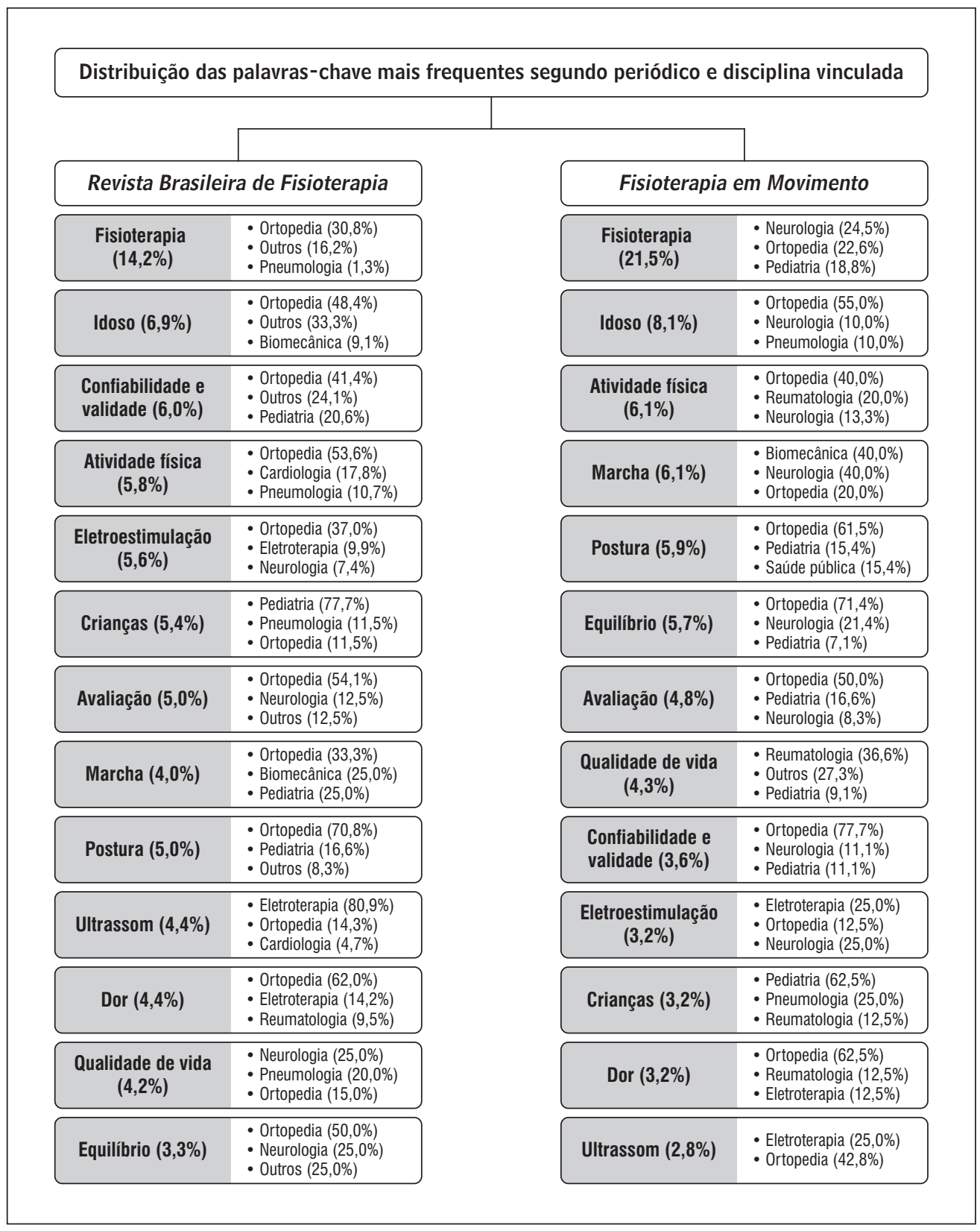

Figura 3 - Organograma representativo da relação entre as palavras-chave mais frequentes e a disciplina vinculada segundo o periódico 
O conhecimento da prática da fisioterapia ainda tem de ser mais estudado e explicado. A maioria das técnicas de tratamento aparentemente continua a ser implícita, tendo professores que as ensinam com base na autoridade e estudantes que aceitam isso e acabam aprendendo por eles mesmos o que seus predecessores já sabiam $(11,12)$. Isso não quer dizer que o tratamento da fisioterapia não é efetivo, apenas que existe pouca evidência do que ele seja (12). Se mais pessoas aptas a fazer pesquisa e publicar seus resultados aparecerem, tanto o corpo de conhecimento como a prática da profissão serão potencialmente desenvolvidos. A fisioterapia como uma profissão clínica deve focar-se diretamente em desenvolver o conhecimento e os métodos de gerar conhecimento que reconheçam isso (12-14).

Tradicionalmente, a fisioterapia adota um modelo de ensino voltado para a transmissão de informação relacionada às técnicas de tratamento, levando o estudante a interessar-se quase que exclusivamente pelas disciplinas técnicas, pois o ensino acaba por valorizar o "como fazer", em vez de buscar respostas do "por que fazer". Assim, as intervenções do fisioterapeuta são assumidas clinicamente como eficazes, porém, muitas vezes, permanecem sem evidências científicas, pois a informação científica não é devidamente valorizada e, por consequência, não aplicada à prática (14-16).

Mesmo após 40 anos de regulamentação a prática fisioterapêutica continua restrita. 0 DecretoLei 938/692 institui como atividade privativa do fisioterapeuta executar métodos e técnicas fisioterápicas com a finalidade de restaurar, desenvolver e conservar a capacidade física do paciente. Tal definição demonstra os pressupostos históricos de preocupação com os métodos e as técnicas e o descaso com a análise e a produção de novos saberes (17).

Ao analisar as áreas de estudo mais prevalentes da publicação em fisioterapia, referente às revistas selecionadas, observa-se a hegemonia da área ortopédica. Nicida (18) aponta que, historicamente, a atuação do fisioterapeuta concentrou-se em hospitais, clínicas de reabilitação e entidades beneficentes sob tutela dos médicos ortopedistas, tanto que as primeiras áreas de atuação foram a ortopedia, com prioridade para as lesões do esporte, e a neurologia, prioritariamente com enfoque em neuropediatria.

Para Caldas (10), aproximadamente até os anos 80 as áreas de atendimento da fisioterapia centravamse na ortopedia, reumatologia, cardiopneumologia e neurologia, cuja ordem é semelhante à encontrada no presente estudo. Corroborando com esses resultados, Coury e Vilella (4), por meio da análise do currículo lattes dos profissionais fisioterapeutas com titulação de doutorado, observaram mais estudos na área ortopédica, seguida das áreas de cardiopneumologia e neurologia. Os autores justificam essa distribuição em razão do doutoramento em fisioterapia prioritariamente nessas áreas.

Diante desses fatos, pode-se suspeitar que o avanço da disciplina "cardiopneumologia" trata-se de uma mudança de paradigma. No entanto, a "ortopedia" mantém-se líder em todas as épocas estudadas e não deve ser julgada negativamente. Conforme Morin (19), a inteligência necessita de certas condições para afirmar-se e desenvolver-se; precisa ser alimentada por acontecimentos e fortalecida por provas; necessita se autorrenovar pelo próprio exercício. Assim, o fôlego da pesquisa em fisioterapia ortopédica deve ser encarado como um processo de desenvolvimento e fortalecimento da disciplina, seguindo como exemplo para as demais.

Sob o olhar dessa problemática, os resultados mostram que a disciplina "saúde pública", vista como de cunho social, foi uma das menos prevalentes em todos os resumos analisados. Para Noronem e Widstrom (6), enquanto o fisioterapeuta não identificar o seu papel e for capaz de falar claramente sobre ele nas equipes multidisciplinares de saúde, a profissão não irá avançar nessa área. Machado (20) reforça essa questão quando coloca que, apesar de o fisioterapeuta ter tradição de trabalho, trata-se de uma profissão nova, com pouca participação nas equipes do Sistema Único de Saúde. Um aspecto que também pode ser levado em conta é o fato de que as disciplinas voltadas para o campo de saúde coletiva nem sempre conseguem despertar no acadêmico o mesmo interesse das demais (21). Esse contexto reflete a pouca expressão da produção de conhecimento em fisioterapia voltada à saúde pública encontrada nesse artigo.

Atualmente, percebe-se que as discussões nas escolas de formação biomédica quebraram algumas amarras e têm sido favoráveis à religação dos saberes e à humanização dos atendimentos, abrindo espaço para a saúde pública. A carência de tratamento mais humanitário e social das pessoas que necessitam do profissional de saúde tem levado a repensar o ensino em toda a área da saúde, buscando a inclusão de novos olhares (22), já que historicamente 
a formação escolar ensina a separar os objetos de seu contexto e as disciplinas umas das outras, dificultando a tentativa de relacioná-las.

A estruturação multidisciplinar da área da saúde vem ampliando o espaço para as discussões dos conceitos de profissão (6). Para Morin, Pena-Veja e Paillard (23), a disciplina é uma categoria organizadora dentro do conhecimento científico e tende naturalmente à autonomia pela delimitação das fronteiras, da linguagem em que ela se constitui, das técnicas que é levada a elaborar e a utilizar e, eventualmente, pelas teorias que lhe são próprias. Dessa forma, ao categorizar os artigos dos periódicos analisados neste estudo em uma única disciplina, limitou-se a noção de interdisciplinaridade. Por exemplo, ao analisar o descritor "criança", observase que não se resumiu somente à disciplina "pediatria", mas também circulou em áreas como a "ortopedia" e a "pneumologia". Essas noções migradoras, assim chamadas por Morin, Pena-Veja e Paillard (23), pressupõem solidariedade dos saberes e contrapõem-se à solidão disciplinar.

A busca por temas relevantes e atuais torna-se um dos maiores desafios a ser superado na realização de pesquisas científicas (24). Por meio desta pesquisa foi possível observar que a produção de conhecimento em fisioterapia segue as tendências do processo de transição demográfica que o País atravessa, resultando na palavra-chave "idoso" como o segundo descritor mais frequente. Os avanços na medicina geriátrica também corroboram para esse resultado.

Para Oliveira et al. (24), com o notável avanço dos estudos em saúde, observa-se um interesse crescente sobre o tema "qualidade de vida", nona palavra-chave mais frequente entre os artigos analisados nesta pesquisa. Assim, esse tema surge como foco imprescindível de pesquisas dessa área e seus resultados tendem a contribuir para a avaliação da relação custo $\mathrm{x}$ benefício do cuidado prestado, auxiliando na definição de tratamentos, além de orientar o acompanhamento ao paciente.

A identificação desses resultados demonstra, por exemplo, o avanço da "pneumologia" e das pesquisas científicas voltadas para uma análise mais humana, como a "qualidade de vida". Assim, é possível imaginar que atualmente ocorre um processo de transição, marcado por um período de crise e indefinição, em que a "ortopedia" permanece hegemônica, mas outras vertentes começam a ganhar espaço.
Por meio da produção de conhecimento em fisioterapia é possível perceber a identidade da profissão. Conforme os resultados do presente estudo, a produção científica em ortopedia apresentou maior ocorrência, refletindo, portanto, a maior área de atuação profissional.

Disseminar os resultados de pesquisas científicas pode refletir na melhora da qualidade do serviço prestado, além projetar cenários futuros (25). A necessidade de operar a crítica interna da produção tem sido apontada como condição para que o conhecimento produzido possa ser incorporado pela comunidade e cumprir o seu papel transformador $(1,26)$. Diante do exposto, torna-se cada vez mais necessária a divulgação de práticas fisioterapêuticas baseadas em evidências (27-30). Uma alternativa para essa situação é a formação de cientistas clínicos para desenvolver o conhecimento relacionado à profissão. Os clínicos devem ser considerados cientistas, e os campos de concentração do conhecimento (universidades) devem se aproximar deles. Assim, essa divisão ciência/prática pode diminuir ao mesmo tempo em que aumenta a produção do conhecimento que é relevante para a prática $(11,12)$.

Cabe, neste momento, a reflexão filosófica: de onde viemos e para onde vamos? Identificando o percurso da produção de conhecimento em fisioterapia é que se pode prever os rumos da profissão.

\section{Referências}

1. Berberian AP, Ferreira LP, Corteletti LCBJ, Azevedo JBM, Marques JM. A produção do conhecimento em distúrbios da comunicação: análise de periódicos (20002005). Rev Soc Bras Fonoaudiol. 2009;14(2):153-9.

2. Rummler G, Silva VR. Longevidade e dispersão física de periódicos nacionais sobre pesquisa, ensino e educação. Educação Temática Digital. 2005;6(2):1-13.

3. Nascimento MC, Sampaio RF, Salmela JH, Mancini MC, Figueiredo IM. A profissionalização da fisioterapia em Minas Gerais. Rev Bras Fisioter. 2006;10(2):241-7.

4. Coury HJCG, Vilella I. Perfil do pesquisador fisioterapeuta brasileiro. Rev Bras Fisioter. 2009;13(4):356-63.

5. Jette DU, Bacon K, Batty C, Carlson M, Ferland A, Hemingway RD, et al. Evidence-based practice: beliefs, attitudes, knowledge, and behaviors of physical therapists. Phys Ther. 2003;83(9):786-805. 
6. Noronem L, Widstrom GC. Professional theoretical paper: towards a paradigm-oriented approach in physical therapy. Physical Therapy Theory and Practice. 1999;15(1):175-84.

7. Bufrem LS, Silva HFN, Fabian CLSRM, Sorribas TV. Produção científica em ciência da informação: análise temática em artigos de revistas brasileiras. Perspectivas em Ciência da Informação. 2007;12(1):38-49.

8. Gil AC. Métodos e técnicas de pesquisa social. 3a ed. São Paulo: Atlas; 1991.

9. Costa D, Nascimento JV. Mudanças no sistema de avaliação dos programas de pós-graduação. Rev Bras Fisioter. 2008;12(4):5-6.

10. Caldas MAJ. 0 processo de profissionalização do fisioterapeuta: o olhar em Juiz de Fora [tese]. Rio de Janeiro: UERJ; 2006.

11. Robertson JV. Research and the cumulation of knowledge in physical therapy. Phys Ther. 1995;75(3):223-32.

12. Robertson JV. Epistemology, private knowledge, and the real problems in physiotherapy. Physiotherapy. 1996;82(9):534-9.

13. Kuhn TS. A estrutura das revoluções científicas. 9a ed. São Paulo: Perspectiva; 2009.

14. Filippin LI, Wagner MB. Fisioterapia baseada em evidência: uma nova perspectiva. Rev Bras Fisioter. 2008; 12(5):432-3.

15. Higgs J, Titchen A. The nature, generation and verification of knowledge. Physiotherapy. 1995;81(9): 521-30.

16. Maher CG, Sherrington C, Elkins M, Herbert RD, Moseleyet AM. Challenges for evidence-based physical therapy: accessing and interpreting high-quality evidence on therapy. Phys Ther. 2004;84(7):644-54.

17. Bispo Júnior JP. Fisioterapia e saúde coletiva: desafios e novas responsabilidades profissionais. Ciênc Saúde Coletiva. 2010;15(Supl. 1):1627-36.

18. Nicida DP. A interdisciplinaridade como um caminho para a formação do fisioterapeuta [dissertação]. São Paulo: UNICID; 2002.

19. Morin E. 0 método 3. Porto Alegre: Sulina; 2009.

20. Machado MH. Formação profissional desestruturada: o desafio da saúde pública. Rev O COFFITO. 2004; 21:15-19.
21. Schwingel GA. Fisioterapia na saúde pública: um agir técnico, político e transformador. In: Barros FBM (Org.). 0 fisioterapeuta na saúde da população: atuação transformadora. Rio de Janeiro: FisioBrasil; 2002.

22. Meyer PF, Costa ICC, Gico VV. Ciências sociais e fisioterapia: uma aproximação possível. Rev Hist Ciênc Saúde. 2006;13(4):877-90.

23. Morin E, Pena-Vega A, Paillard B. Diálogo sobre o conhecimento. São Paulo: Cortez; 2004.

24. Oliveira CJ, Pereira CAR, Pontes JNC, Melo Filho AV, Moreira TMM. Análise da produção científica na área da saúde sobre qualidade de vida no Brasil entre 2000 e 2005: um estudo bibliográfico. Rev Eletr Enf. 2007;9(2):496-505.

25. Kumar SP. Sorting out lemons and oranges: towards better quality of reporting clinical trials in Journal of Physical Therapy - The CONSORT 2010 Statement. J Phys Ther. 2010;1:1-10.

26. Castro MLS. Educação e economia: análise de artigos publicados em periódicos brasileiros: 1982-2000. Análise. 2006;17(2):224-33.

27. Maher CG, Moseley AM, Sherrington C, Eikins MR, Herbert RD. A description of the trials, reviews, and practice guidelines indexed in the PEDro database. Phys Ther. 2008;88(9):1068-77.

28. Elkins M, Ada L. Quality of trials in Australian Journal of Physiotherapy. Aust J Physiother. 2009; 55(4):233-4.

29. Moseley AM, Herbert RD, Sherrington C, Maher CG. Evidence for physiotherapy practice: a survey of the Physiotherapy Evidence Database (PEDro). Aust J Physiother. 2002;48(1):43-9.

30. Marques AP, Peccin MS. Pesquisa em fisioterapia: a prática baseada em evidências e modelos de estudos. Fisioter Pesq. 2005;1(1):43-8.

Recebido: 31/05/2010 Received: 05/31/2010

Aprovado: 18/11/2010 Approved: 11/18/2010 